\title{
Quality of Life in Patients Treated with Palliative Radiotherapy for Advanced Lung Cancer and Lung Metastases
}

\author{
Kaitlin $\mathrm{Koo}^{\mathrm{a}}$, Liang Zeng ${ }^{\mathrm{a}}$, Florencia Jon ${ }^{\mathrm{a}}$, Emily Chen ${ }^{\mathrm{a}}$, Kristopher Dennis ${ }^{\mathrm{a}}$, Lori Holden ${ }^{\mathrm{a}}$, \\ Liying Zhanga, Amanda Caissie ${ }^{\mathrm{a}}$, Janet Nguyen ${ }^{\mathrm{a}}$, May Tsao ${ }^{\mathrm{a}}$, Elizabeth Barnes ${ }^{\mathrm{a}}$, \\ Cyril Danjoux ${ }^{\mathrm{a}}$, Arjun Sahgal ${ }^{\mathrm{a}}$, Edward Chow ${ }^{\mathrm{a}, \mathrm{b}}$
}

\begin{abstract}
Background: The purpose of this study was to investigate quality of life (QOL) in patients receiving palliative radiotherapy (RT) for advanced lung cancer/lung metastases using the EORTC QLQLC13 and the EORTC QLQ-C15-PAL questionnaires.
\end{abstract}

Methods: Patients who received palliative RT for lung metastases or advanced lung cancer between November 2007 and October 2010 completed the EORTC QLQ-LC13 and the QLQ-C15-PAL at baseline prior to RT, 1, 2, 4, 8 and 12 weeks post-treatment. The Wilcoxon Signed Rank test was used to compare QOL scores between baseline and each follow-up period.

Results: Thirty-one patients with advanced lung disease were included in this study; $61 \%$ of participants were male and $39 \%$ were female. The median age was 69 years (range 38 - 85), and median KPS and PPS scores at baseline were both 70 (range 30 - 90). All patients received radiotherapy to the lung. None of the QLQ-LC13 scores significantly improved or deteriorated at any follow-up. Of the QLQ-C15-PAL scales, fatigue, pain, insomnia and physical functioning significantly improved at their respective follow-ups.

Conclusions: This was the first study to use the EORTC QLQLC13 in conjunction with the EORTC QLQ-C15-PAL questionnaires. Future studies should continue to incorporate quality of life assessment tools specific to disease characteristics in advanced cancer patients.

Manuscript accepted for publication March 11, 2011

${ }^{a}$ Rapid Response Radiotherapy Program, Department of Radiation Oncology, Odette Cancer Center, Sunnybrook Health Sciences Center, University of Toronto, Toronto, Ontario, Canada

${ }^{\mathrm{b}}$ Corresponding author: Edward Chow, Department of Radiation Oncology, Odette Cancer Center, Sunnybrook Health Sciences Center, 2075 Bayview Avenue, Toronto, ON, M4N3M5, Canada.

Email: Edward.Chow@sunnybrook.ca

doi:10.4021/wjon288w
Keywords: QLQ-C15-PAL; QLQ-LC13; Quality of life; Advanced cancer; Radiotherapy; Lung; Metastases

\section{Introduction}

Patients with advanced lung cancer or lung metastases (herein referred to as advanced lung disease) often present with thoracic symptoms such as hemoptysis, cough, chest pain, dysphagia and dyspnea. In the primary or non-metastatic setting, fewer than $15 \%$ of patients achieve long-term survival $[1,2]$. Treatment intent is often palliative and health-related quality of life (QOL) becomes the primary treatment goal. Although patients' self-reported QOL is a good indicator of overall well-being, it has infrequently been investigated in patients with advanced lung disease [3-9].

Palliative radiotherapy (RT) is effective in ameliorating symptoms experienced by patients with advanced lung disease [9-11] and has been shown to improve or at least preserve QOL [8]. There is limited literature that investigates QOL after treatment with palliative RT using validated lung symptom-specific tools.

The European Organization for Research and Treatment of Cancer (EORTC) addressed the need for standardized QOL tools by coordinating the development of both a generalized QOL questionnaire and disease-specific QOL questionnaires. The purpose of this study was to use the QLQC15-PAL and the QLQ-LC13 to investigate the effectiveness of RT in improving the QOL of patients receiving palliative radiotherapy for advanced lung disease.

\section{Methods}

The Rapid Response Radiotherapy Program (RRRP) at the Odette Cancer Center, Sunnybrook Health Sciences Center, Toronto, Ontario, Canada, provides timely access to palliative RT for patients with advanced cancer. Patients referred to the RRRP between November 2007 and October 2010, receiving palliative radiotherapy for symptomatic advanced 
primary lung cancer or lung metastases were eligible for this study. Patients accrued to this study had evidence of malignancy, radiological evidence of primary lung cancer or metastases to the lung, spoke English and provided informed and written consent. Baseline information collected included age, gender, primary cancer site, Karnofsky Performance Status (KPS), and Palliative Performance Scale (PPS) score. Health-related QOL was assessed using the EORTC QLQC15-PAL and QLQ-LC13. Patients completed both questionnaires at baseline, prior to RT, and weeks 1, 2, 4, 8 and 12 from the start of RT. Baseline questionnaires were conducted in person and a trained research assistant completed subsequent telephone follow-up questionnaires. All research was conducted following approval from the Sunnybrook Health Science Center research ethics board.

\section{The EORTC QLQ-C15-PAL}

The QLQ-C15-PAL is an abbreviated version of the commonly used EORTC QLQ-C30 [12]. The QLQ-C15-PAL consists of 15 questions: five items assessing physical and emotional functioning, four items assessing fatigue and pain, five item symptom scales (nausea/vomiting, dyspnea, insomnia, appetite loss, constipation) and one final question assessing overall QOL $[13,14]$.

\section{The EORTC QLQ-LC13}

The EORTC QLQ-LC13 is a supplementary questionnaire for patients with lung cancer and includes questions assessing cough, hemoptysis, dyspnea, site-specific pain, treatment-related side effects (sore mouth, dysphasia, peripheral neuropathy and alopecia) and the efficacy of pain medications [15].

\section{Statistical analysis}

The Wilcoxon Signed Rank test was used to determine changes in QLQ-C15-PAL and QLQ-LC13 scores between baseline and each follow-up visit (i.e., week 1, week 2, month 1, month 2, and month 3). All analyses were conducted by Statistical Analysis Software (SAS version 9.2 for Windows). Two-sided P-values of less than or equal to 0.05 were considered statistically significant.

\section{Results}

\section{Patient demographics}

A total of 31 patients were enrolled. Patient demographics are shown in Table 1. Sixty-one percent of participants were male and thirty-nine percent were female. Their median age was 69 years (range 38 - 85), and their median KPS
Table 1. Patients Demographics $(N=31)$

\begin{tabular}{|c|c|c|}
\hline \multicolumn{3}{|l|}{ Age (years) } \\
\hline $\mathrm{n}$ & \multicolumn{2}{|l|}{31} \\
\hline Mean \pm SD & \multicolumn{2}{|c|}{$68 \pm 11$} \\
\hline Inter-quartiles & \multicolumn{2}{|c|}{$58-77$} \\
\hline Median (range) & \multicolumn{2}{|c|}{$69(38-85)$} \\
\hline \multicolumn{3}{|c|}{ Karnofsky Performance Scale } \\
\hline $\mathrm{n}$ & \multicolumn{2}{|l|}{31} \\
\hline Mean \pm SD & \multicolumn{2}{|c|}{$67 \pm 16$} \\
\hline Inter-quartiles & \multicolumn{2}{|c|}{$60-80$} \\
\hline Median (range) & \multicolumn{2}{|c|}{$70(30-90)$} \\
\hline \multicolumn{3}{|c|}{ Palliative Performance Scale } \\
\hline $\mathrm{n}$ & \multicolumn{2}{|l|}{30} \\
\hline Mean \pm SD & \multicolumn{2}{|c|}{$65 \pm 17$} \\
\hline Inter-quartiles & \multicolumn{2}{|c|}{$60-80$} \\
\hline Median (range) & \multicolumn{2}{|c|}{$70(30-90)$} \\
\hline \multicolumn{3}{|l|}{ Gender } \\
\hline Male & 19 & $(61 \%)$ \\
\hline Female & 12 & $(39 \%)$ \\
\hline \multicolumn{3}{|l|}{ Primary Cancer Site } \\
\hline Lung & 25 & $(80 \%)$ \\
\hline GI-Colon & 2 & $(6 \%)$ \\
\hline GI-Rectum & 1 & $(3 \%)$ \\
\hline Breast & 1 & $(3 \%)$ \\
\hline Prostate & 1 & $(3.23 \%)$ \\
\hline Renal Cell & 1 & $(3 \%)$ \\
\hline
\end{tabular}

and PPS scores at baseline were both 70 (ranges 30 - 90). At weeks 1, 2, 4, 8 and 12 weeks, 14 (45\%), 14 (45\%), 15 (48\%), 12 (39\%) and 9 (29\%) patients completed followup questionnaires, respectively. The most common primary cancers were of the lung (80\%) and colon (6\%).

\section{QLQ-C15-PAL score comparisons between baseline and each follow-up visit}

Fatigue, pain, insomnia and physical functioning significantly improved from baseline at different time points during follow-up (Fig. 1). Fatigue significantly improved at week 1 when compared to baseline $(\mathrm{P}=0.04)$. Pain significantly improved at week $2(\mathrm{P}=0.03)$. Insomnia improved at months $1(\mathrm{P}=0.008)$ and $2(\mathrm{P}=0.05)$ while physical functioning 


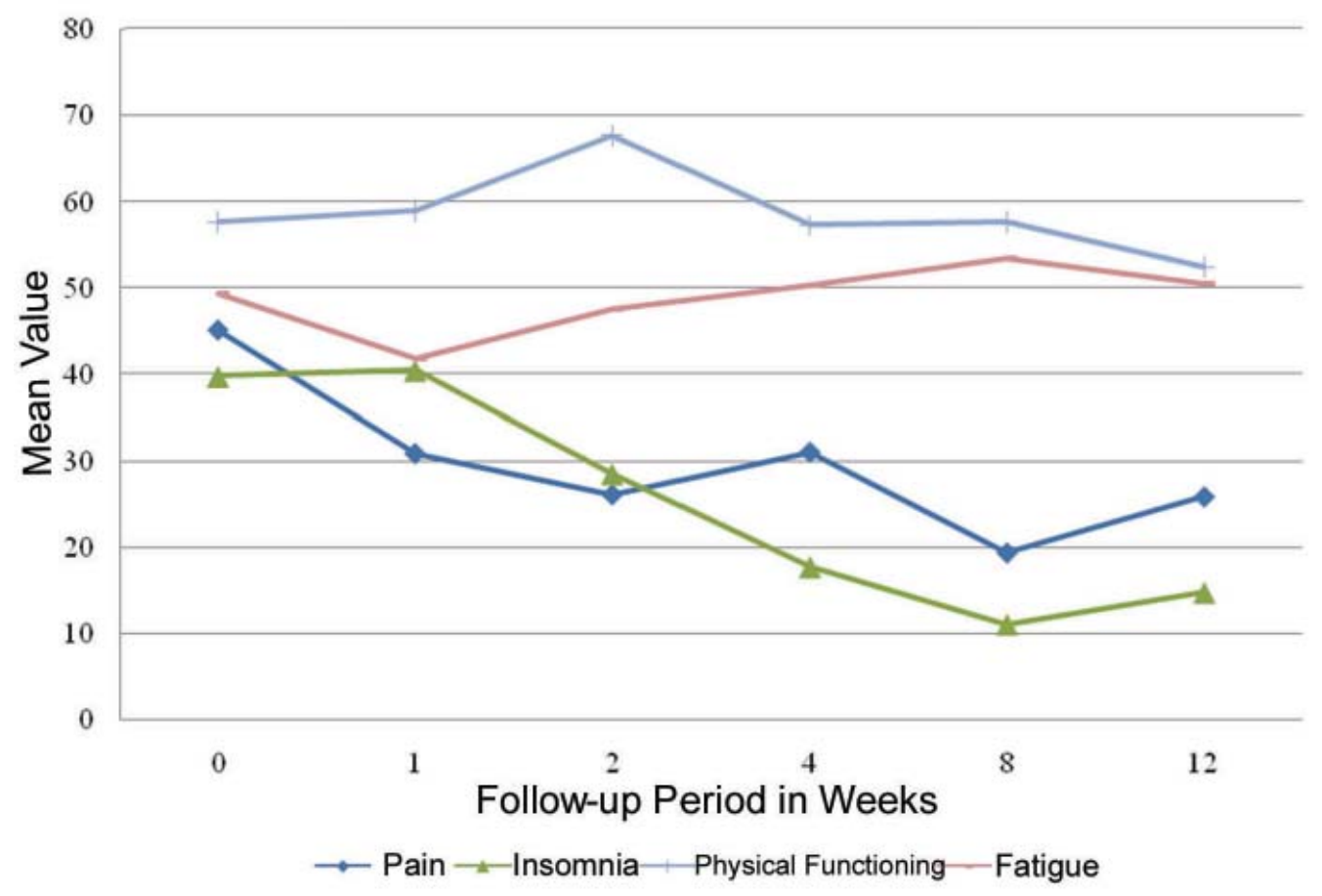

Figure 1. QLQ-C15-PAL scores with significant differences between follow-up and baseline.

Table 2. QLQ-C15-PAL Mean Score Value for Each Symptom Item at Baseline and Follow-up

\begin{tabular}{lllllll}
\hline \multirow{2}{*}{ Symptom Scales/Items } & \multicolumn{5}{c}{ Data Collection Period in Weeks } \\
\cline { 2 - 7 } & $\mathbf{0}$ & $\mathbf{1}$ & $\mathbf{2}$ & $\mathbf{4}$ & $\mathbf{8}$ & $\mathbf{1 2}$ \\
\hline Pain & 45.16 & 30.95 & $\mathbf{2 6 . 1 9}$ & 31.11 & 19.44 & 25.93 \\
Dyspnea & 45.56 & 42.86 & 30.95 & 48.89 & 33.33 & 40.74 \\
Insomnia & 39.78 & 40.48 & 28.57 & $\mathbf{1 7 . 7 8}$ & $\mathbf{1 1 . 1 1}$ & 14.81 \\
Appetite Loss & 41.11 & 38.1 & 23.81 & 31.11 & 33.33 & 59.26 \\
Constipation & 30 & 28.57 & 21.43 & 24.44 & 16.67 & 40.74 \\
Overall Quality of Life & 51.67 & 54.76 & 50 & 56.67 & 52.78 & 55.56 \\
Physical Functioning & 57.7 & 59.05 & 67.69 & 57.33 & 57.78 & $\mathbf{5 2 . 5}$ \\
Fatigue & 49.46 & $\mathbf{4 1 . 8 8}$ & 47.62 & 50.37 & 53.54 & 50.62 \\
Nausea/Vomiting & 12.9 & 15.48 & 11.9 & 8.89 & 15.28 & 12.96 \\
Emotional Functioning & 74.14 & 75.6 & 85.12 & 82.22 & 75 & 64.81 \\
\hline
\end{tabular}

QLQ-C15-PAL scores were compared between baseline and at each follow-up visit. Scores that show significant differences at the indicated follow-up period are bolded. Significant differences were calculated using Wilcoxon Signed Rank. 
Table 3. QLQ-LC13 Mean Score Values for Each Symptom Item at Baseline and Follow-up

\begin{tabular}{lllllll}
\hline \multirow{2}{*}{ Symptom Scales/Items } & \multicolumn{7}{c}{ Data Collection Period in Weeks } \\
\cline { 2 - 7 } & $\mathbf{0}$ & $\mathbf{1}$ & $\mathbf{2}$ & $\mathbf{4}$ & $\mathbf{8}$ & $\mathbf{1 2}$ \\
\hline Coughing & 49.46 & 42.86 & 45.24 & 42.22 & 36.11 & 25.93 \\
Hemoptysis & 16.13 & 16.67 & 5.13 & 4.44 & 8.33 & 11.11 \\
Sore Mouth & 6.45 & 7.69 & 4.76 & 6.67 & 8.33 & 7.41 \\
Dysphagia & 16.13 & 19.05 & 9.52 & 15.56 & 5.56 & 3.7 \\
Peripheral Neuropathy & 18.89 & 26.19 & 0 & 17.78 & 5.56 & 11.11 \\
Alopecia & 8.89 & 0 & 7.69 & 28.89 & 30.3 & 37.5 \\
Pain in Chest & 27.96 & 30.95 & 26.19 & 15.56 & 19.44 & 22.22 \\
Pain in Arm & 22.58 & 16.67 & 9.52 & 24.44 & 5.56 & 18.52 \\
Pain Other & 39.78 & 25.64 & $\mathbf{1 2 . 8 2}$ & 26.67 & 30.56 & 44.44 \\
Dyspnea & 30.95 & 33.33 & 16.67 & 30.56 & 26.39 & 36.51 \\
Dsypnea When Rested & 2.33 & 1.5 & 2.25 & 2 & 1.33 & 2.5 \\
Dyspnea When Walked & 2.33 & 2 & 2.75 & 2 & 1.67 & 3 \\
\hline
\end{tabular}

QLQ-LC13 scores were compared between baseline and at each follow-up visit. Scores with a significant difference at the indicated follow-up period are bolded. Significant differences were calculated using Wilcoxon Signed Rank.

improved at month 3 compared to baseline $(\mathrm{P}=0.05)$. There were no significant differences or worsening of symptoms between any of the other items after receiving RT (Table 2).

\section{QLQ-LC13 score comparisons between baseline and each follow-up visit}

Coughing, hemoptysis, sore mouth, dysphagia, peripheral neuropathy, alopecia, pain in the chest, pain in the arm and dyspnea did not significantly change during any follow-up period (Table 3). The score for 'other pain' significantly improved at week 2 when compared to baseline $(\mathrm{P}=0.02)$.

\section{Discussion}

To our knowledge, this was the first study to use the EORTC QLQ-C15-PAL in conjunction with the EORTC QLQ-LC13 to investigate QOL in patients with advanced lung disease receiving palliative radiotherapy. The use of the QLQ-LC13 and the QLQ-C15-PAL are ideal in this setting due to their brevity, site- and patient-specific focus. In our study, we found 'other pain' was the only lung specific symptom that improved post-radiotherapy as assessed by the QLQ-LC13.
However due to variability associated with the specific area of pain for this item, we cannot confidently include this within our findings. Pain, insomnia, physical functioning and fatigue, as measured by the QLQ-C15-PAL significantly improved at some period after treatment.

Fairchild et al. performed a meta-analysis investigating optimum palliative RT for advanced stage lung cancers [9], suggesting that both high and low RT dose schedules provided some level of improvement in thoracic symptoms secondary to lung cancer. As a reflection of the limited available literature, validated QOL data was compiled as part of the aforementioned analysis and identified as an area of research requiring further investigation. The authors indicated the need to include QOL as a meaningful endpoint when evaluating treatments for advanced lung disease; a sentiment echoed by other authors as well [7, 8, 13, 16-23].

Salvo et al. reviewed QOL assessment tools for patients receiving palliative radiotherapy for advanced lung cancer and lung metastases. This review encouraged investigators to include validated, specific QOL instruments such as the EORTC QLQ-LC13 or the FACT-L due to the specificity of these instruments in measuring lung-cancer specific symptoms [24].

Other studies assessing QOL in patients treated with 
palliative radiotherapy for advanced lung disease have used various other assessment tools such as the Spitzer QLQ Index, Hospital Anxiety and Depression Scale (HADS), Rotterdam Symptom Checklist (RSCL), study-designed QLQ questionnaires, Functional Assessment of Cancer TherapyLung/General (FACT-L/FACT-G), Lung Cancer Symptom Scale, and the EORTC QLQ-LC17 [24]. Salvo et al. suggested that use of validated, lung-specific tools (the FACT-L or EORTC QLQ-LC13) would allow for easier comparisons between trials and would also increase the internal validity of individual studies [24].

Several studies have used the QLQ-LC13 and the QLQC30 when assessing QOL in patients with lung cancer $[8,15$, 19, 22, 23, 25]. Similarly, our study included patients with primary lung cancers and patients with lung metastases. The QLQ-C15-PAL was able to identify improvements in pain, insomnia, fatigue and physical functioning post-RT. These results are consistent with the findings of Hicsonmez, Bezjak and Langendijk [8, 20, 21]. Improvements in insomnia, fatigue and pain may result since each of these symptoms may contribute to the maintenance of the others, resulting in a significant adverse impact on QOL [26].

It is interesting to note that in our study, none of the lung specific symptoms assessed by the QLQ-LC13 significantly improved or deteriorated during any follow-up period. However, palliative radiotherapy may have played a stabilizing role. Hicsonmez et al. found that dyspnea significantly improved post-treatment and Langendijk et al. reported that palliative radiotherapy was effective in palliation of hemoptysis, chest pain and cough as assessed by the QLQC30 and QLQ-LC13 [8, 20]. Furthermore, Lutz et al. found statistically significant improvements in cough, hemoptysis and dyspnea using the Lung Cancer Symptom Scale [7]. Literature exists suggesting that symptoms experienced by this group actually worsened during and immediately following $\mathrm{RT}$, and then returned to baseline levels [13, 14, 16, 19].

Due to the progression of the patient's disease and limited prognosis, many patients were subsequently lost to follow-up. Our analysis of QOL and symptom trends may be more reflective of patients with better prognosis and may not truly represent this population. Radiation therapy for treatment of advanced lung disease is a well-tolerated therapeutic modality and preserves QOL. As the goals of treatment shift from survival to QOL, specific assessment tools such as the QLQ-LC13 should be incorporated in future clinical trials investigating patients with lung metastases or advanced lung cancer. More efforts should be directed towards investigating the outcomes of those patients who were lost to follow-up, as this was a common limitation expressed by similar studies.

\section{Acknowledgements}

This study was generously supported by the Michael and
Karyn Goldstein Cancer Research Fund. The authors thank Mrs. Stacy Yuen for her administrative assistance.

\section{Conflicts of Interest}

None

\section{References}

1. Ozturk A, Sarihan S, Ercan I, Karadag M. Evaluating quality of life and pulmonary function of long-term survivors of non-small cell lung cancer treated with radical or postoperative radiotherapy. Am J Clin Oncol 2009;32(1):65-72.

2. Parkin DM. Global cancer statistics in the year 2000 . Lancet Oncol 2001;2(9):533-543.

3. Muers MF, Round CE. Palliation of symptoms in nonsmall cell lung cancer: a study by the Yorkshire Regional Cancer Organisation Thoracic Group. Thorax 1993;48(4):339-343.

4. Inoperable non-small-cell lung cancer (NSCLC): a Medical Research Council randomised trial of palliative radiotherapy with two fractions or ten fractions. Report to the Medical Research Council by its Lung Cancer Working Party. Br J Cancer 1991;63(2):265-270.

5. A Medical Research Council (MRC) randomised trial of palliative radiotherapy with two fractions or a single fraction in patients with inoperable non-small-cell lung cancer (NSCLC) and poor performance status. Medical Research Council Lung Cancer Working Party. Br J Cancer 1992;65(6):934-941.

6. Rees GJ, Devrell CE, Barley VL, Newman HF. Palliative radiotherapy for lung cancer: two versus five fractions. Clin Oncol (R Coll Radiol) 1997;9(2):90-95.

7. Lutz ST, Huang DT, Ferguson CL, Kavanagh BD, Tercilla OF, Lu J. A retrospective quality of life analysis using the Lung Cancer Symptom Scale in patients treated with palliative radiotherapy for advanced nonsmall cell lung cancer. Int J Radiat Oncol Biol Phys 1997;37(1):117122.

8. Langendijk JA, ten Velde GP, Aaronson NK, de Jong JM, Muller MJ, Wouters EF. Quality of life after palliative radiotherapy in non-small cell lung cancer: a prospective study. Int J Radiat Oncol Biol Phys 2000;47(1):149-155.

9. Fairchild A, Harris K, Barnes E, Wong R, Lutz S, Bezjak A, Cheung $P$, et al. Palliative thoracic radiotherapy for lung cancer: a systematic review. J Clin Oncol 2008;26(24):4001-4011.

10. Efficace F, Bottomley A, Smit EF, Lianes P, Legrand C, Debruyne C, Schramel F, et al. Is a patient's self-reported health-related quality of life a prognostic factor for survival in non-small-cell lung cancer patients? A mul- 
tivariate analysis of prognostic factors of EORTC study 08975. Ann Oncol 2006;17(11):1698-1704.

11. Brundage MD, Bezjak A, Dixon P, Grimard L, Larochelle $\mathrm{M}$, Warde $\mathrm{P}$, Warr $\mathrm{D}$. The role of palliative thoracic radiotherapy in non-small cell lung cancer. Can $\mathrm{J}$ Oncol 1996;6 Suppl 1:25-32.

12. Groenvold M, Petersen MA, Aaronson NK, Arraras JI, Blazeby JM, Bottomley A, Fayers PM, et al. The development of the EORTC QLQ-C15-PAL: a shortened questionnaire for cancer patients in palliative care. Eur $\mathrm{J}$ Cancer 2006;42(1):55-64.

13. Pijls-Johannesma M, Houben R, Boersma L, Grutters J, Seghers K, Lambin P, Wanders R, et al. High-dose radiotherapy or concurrent chemo-radiation in lung cancer patients only induces a temporary, reversible decline in QoL. Radiother Oncol 2009;91(3):443-448.

14. John LD. Quality of life in patients receiving radiation therapy for non-small cell lung cancer. Oncol Nurs Forum 2001;28(5):807-813.

15. Chie WC, Yang CH, Hsu C, Yang PC. Quality of life of lung cancer patients: validation of the Taiwan Chinese version of the EORTC QLQ-C30 and QLQ-LC13. Qual Life Res 2004;13(1):257-262.

16. Auchter RM, Scholtens D, Adak S, Wagner H, Cella DF, Mehta MP. Quality of life assessment in advanced nonsmall-cell lung cancer patients undergoing an accelerated radiotherapy regimen: report of ECOG study 4593. Eastern Cooperative Oncology Group. Int J Radiat Oncol Biol Phys 2001;50(5):1199-1206.

17. Ostlund U, Wennman-Larsen A, Gustavsson P, Wengstrom Y. What symptom and functional dimensions can be predictors for global ratings of overall quality of life in lung cancer patients? Support Care Cancer 2007;15(10):1199-1205.

18. Turner NJ, Muers MF, Haward RA, Mulley GP. Do elderly people with lung cancer benefit from palliative radiotherapy? Lung Cancer 2005;49(2):193-202.
19. Sundstrom S, Bremnes R, Brunsvig P, Aasebo U, Olbjorn K, Fayers PM, Kaasa S. Immediate or delayed radiotherapy in advanced non-small cell lung cancer (NSCLC)? Data from a prospective randomised study. Radiother Oncol 2005;75(2):141-148.

20. Hicsonmez A, Kose K, Andrieu MN, Guney Y, Kurtman C. The European Organization for Research and Treatment of Cancer core quality of life questionnaire (QLQ-C30 version 3.0 Turkish) in cancer patients receiving palliative radiotherapy. Eur J Cancer Care (Engl) 2007;16(3):251-257.

21. Bezjak A, Dixon P, Brundage M, Tu D, Palmer MJ, Blood P, Grafton C, et al. Randomized phase III trial of single versus fractionated thoracic radiation in the palliation of patients with lung cancer (NCIC CTG SC.15). Int J Radiat Oncol Biol Phys 2002;54(3):719-728.

22. Langendijk H, de Jong J, Tjwa M, Muller M, ten Velde G, Aaronson N, Lamers R, et al. External irradiation versus external irradiation plus endobronchial brachytherapy in inoperable non-small cell lung cancer: a prospective randomized study. Radiother Oncol 2001;58(3):257-268.

23. Langendijk JA, Aaronson NK, ten Velde GP, de Jong JM, Muller MJ, Wouters EF. Pretreatment quality of life of inoperable non-small cell lung cancer patients referred for primary radiotherapy. Acta Oncol 2000;39(8):949958.

24. Salvo N, Hadi S, Napolskikh J, Goh P, Sinclair E, Chow E. Quality of life measurement in cancer patients receiving palliative radiotherapy for symptomatic lung cancer: a literature review. Curr Oncol 2009;16(2):16-28.

25. Nicklasson M, Bergman B. Validity, reliability and clinical relevance of EORTC QLQ-C30 and LC13 in patients with chest malignancies in a palliative setting. Qual Life Res 2007;16(6):1019-1028.

26. Theobald DE. Cancer pain, fatigue, distress, and insomnia in cancer patients. Clin Cornerstone 2004;6 Suppl 1D:S15-21. 